\title{
PTA-DESTINATION: \\ a decision support system to ensure human and environmental protection
}

\author{
M. Orso Giacone ${ }^{1}$, A. Navarretta ${ }^{2} \&$ F. Bratta ${ }^{1}$ \\ ${ }^{1}$ Environment and Territory Directorate, Regione Piemonte, Italy \\ ${ }^{2}$ CSI-Piemonte, Consortium for Information Systems, Italy
}

\begin{abstract}
"PTA-DESTINATION. Shared cross border routes", funded by the Italy/Switzerland Operational Programme for Trans-frontier Cooperation 20072013 , is a project whose objective is to capitalize the result of the previous projects PTA (Alpine Technological Platform) and DESTINATION (DangErous tranSport To New prevenTive Instruments).

The partnership includes Regione Lombardia, as project leader, Università della Svizzera Italiana, Regione Piemonte, Regione Autonoma della Valle d'Aosta and Provincia Autonoma di Bolzano.

Technical collaborators who worked on the DESTINATION project were CSI Piemonte, 5T S.r.l. and Laboratorio Mobilità e Trasporti of Politecnico di Milano.

The main result of the DESTINATION project, which ended in May 2014, is the definition of a risk analysis model of dangerous goods transport (DGT) by road, which constitutes the basis to a shared information system called GIIS (Global Integrated Information System), including environmental, territorial and technical data relevant to meeting local authorities' and private stakeholders' needs:

- supporting decision making processes and guideline definition related to DGT (preventive safety);

- assisting DGT companies through real-time monitoring (active safety);

- mitigating human and environmental impacts in the case of accidents with specific functionalities aimed at emergency assessment and management (passive safety).
\end{abstract}


The paper will give a short overview of the risk analysis model developed in the DESTINATION project and will describe the main features of the GIIS and the ongoing activities of methodology fine tuning and GIIS performance optimization that are being developed within the project PTA-DESTINATION.

Keywords: hazardous materials, dangerous goods transport, risk analysis, environmental risk, social risk, geographic information system, decision support system.

\section{Introduction}

Dangerous Goods Transport by road represents about 5-6\% of the entire amount of transported goods in Europe and, according to the laws and regulations in force, it is not required to monitor and control the route of vehicles transporting hazardous materials.

In recent years more attention has been paid to monitoring transport of dangerous goods by road (Benza et al. [1]) and to prevent risk and manage crisis situations (Pastorelli [2]).

ADR is the European Agreement concerning the International Carriage of Dangerous Goods by Road [3], whose aim is to guarantee a high level of safety in all transport operations, focusing its attention on the requirements road vehicle carrying dangerous goods have to be compliant with and on the driver training. There is, on the contrary, nothing specific on the issue of the vehicleinfrastructure-environment interaction.

DESTINATION project (VV.AA. [4]) started up from the awareness of this lack in legislation with the overall objective to create an instrument and a methodology useful to enrich knowledge on DGT and on the anthropic and environmental vulnerability, in order to ensure human and environmental protection.

Another relevant activity performed has been the identification of the most critical points to concentrate on the monitoring of dangerous goods traffic. A monitoring network of 30 gates able to recognize ADR license plates has been implemented (VV.AA. [5]).

The so-called GIIS processes a risk map of all the area of the project able to display the risk at a local scale, depending on the user needs. Simulation features are also available according to the needs of public and private stakeholders.

The PTA-DESTINATION project wants to enrich the dataset available on the platform developed in the previous project PTA, in order to fine-tune the risk analysis methodology and to optimize GIIS functionalities. One of the aim of the PTA-DESTINATION project is also to implement some Mobile Applications to visualize DGT risk maps and to evaluate the damage areas in case of accident to support emergency assessment and management. 


\section{DGT risk analysis model}

The main problem in DGT risk assessment is that it is an active risk and that the accident location is unpredictable. The point where a hypothetical accident could happen as well as the vulnerabilities in the surrounding areas are unknown.

DESTINATION project developed a Global Integrated Information System (GIIS) for data acquisition on dangerous goods transport by road, on anthropic and environmental vulnerabilities and on the resilience of territory that constitutes the input data set for the calculation of the risk associated to the land transportation of hazardous materials.

The risk analysis model defines different scenarios depending on the accident type and on the dangerous goods substances transported (VV.AA. [6]).

The Risk Formula has been developed after a detailed survey on data availability in the information systems of each partner and after the analysis and sharing between all partners of the factors of major interest for DGT Risk assessment. The parameters composing the formula and their functional dependencies have been defined also taking into account the need of optimizing the implementation of the algorithm in the GIIS.

To determine the connection of each parameter to the elements of major interest, 4 "subscripts" have been defined:

- $\operatorname{arch}$ road (i)

- ADR substance ( $\mathrm{j}$ )

- incident scenario with a specific threshold and a consequential damage area $(\mathrm{k})$

- type and susceptibility of targets (m).

The general risk formula is the following:

$$
R_{i}=P_{i s, i} \times \sum_{j}\left(P_{A D R, i j} \times \sum_{k}\left(P_{s c, i j k} \times \sum_{m}\left(F_{p, m} \times E_{i k m} \times S_{k m} \times\left(1-C_{f f, i k m}\right)\right)\right)\right)
$$

where

$\mathrm{R}_{\mathrm{i}}=\quad$ risk for the community (social/ environmental) in reference to the $i$-th arc [deaths/arc/year] [ $\mathrm{m}^{2} \mathrm{eq}$ with damage/arc/year]

$\mathrm{P}_{\mathrm{is}, \mathrm{I}}=\quad$ intrinsic road danger in reference to the $i$-th arc [circulating vehicles in accidents/arc/ year]

$\mathrm{P}_{\mathrm{ADR}, \mathrm{ij}}=$ occurrence probability of a car accident involving a $j$-th ADR substance in reference to the $i$-th arch [ADR vehicles involved in accidents/circulating vehicles involved in accidents]

$\mathrm{P}_{\mathrm{sc}, \mathrm{j} \mathrm{jk}}=\quad$ occurrence probability of a $k$-th incident scenario with known damage area and threshold $(k)$, (involving the $j$-th substance), in reference to the $i$-th arch [accident events/ADR vehicles involved in accident]

$\mathrm{F}_{\mathrm{p}, \mathrm{m}}=$ presence factor/weight of the $m$-th target potentially exposed [population equivalent present/population equivalent exposed][ $\mathrm{m}^{2} \mathrm{eq}$ $/ \mathrm{m}^{2}$ exposed]

$\mathrm{E}_{\mathrm{ikm}}=m$-th target potentially exposed to the $k$-th accident scenario with known damage area and threshold (involving the $j$-th substance) in reference to the $i$-th arc [population equivalent exposed/accident event $]\left[\mathrm{m}^{2}\right.$ exposed/accident event $]$ 
$\mathrm{S}_{\mathrm{km}}=\quad$ susceptibility of the $m$-th target potentially present at the $k$-th accident scenario with known damage area and threshold [deaths/equivalent inhabitants], [damaged $\mathrm{m}^{2}$ eq/exposed $\mathrm{m}^{2} \mathrm{eq}$ ]

$\mathrm{C}_{\mathrm{ff}, \mathrm{ikm}}=\quad$ ability to address in relation to the $m$-th target potentially exposed to the $k$-th accident scenario with known damage area and threshold (involving the $j$-th substance) in reference to the $i$-th arc [-]

It is important to point out that the formula is structured as a sequence of additions and multiplications, in order to simplify the implementation in the GIIS and to give the possibility to break it down in "prime factors", being able to have as a result not only the cumulated risk on the examined area, but also some intermediate results (see Table 1).

Table 1: Partial formulation of the risk model adopted with important practical uses.

\begin{tabular}{|c|c|}
\hline Definition & Potential purposes \\
\hline \multicolumn{2}{|c|}{ Indicators involving a further specific simulation calculation } \\
\hline$P_{i s, i} \times \sum_{j}\left(P_{A D R, i j} \times \sum_{k}\left(P_{s c, i j k} \times S_{k m} \times\left(1-C_{f f, i k m}\right)\right)\right)$ & $\begin{array}{l}\text { Risk acceptability } \\
\text { for a target } m \\
\text { (extremely useful } \\
\text { for territorial } \\
\text { planning) }\end{array}$ \\
\hline$P_{i s, i} \times \sum_{j}^{\text {F2. Accident probability }}\left(P_{A D R, i j} \times \sum_{k} P_{s c, i j k}\right)$ & $\begin{array}{l}\text { Analysis of ADR } \\
\text { hazard ratings on a } \\
\text { road arc }\end{array}$ \\
\hline $\begin{array}{l}\text { F3. Potentially exposed targets (assumption } \boldsymbol{m} \text { ) } \\
\qquad E_{i k m}\end{array}$ & $\begin{array}{l}\text { Representation of } \\
\text { exposed human and } \\
\text { non-human targets }\end{array}$ \\
\hline \multicolumn{2}{|c|}{$\begin{array}{l}\text { Elementary indicators involving straightforward visualisation } \\
\text { (without further simulation calculations) }\end{array}$} \\
\hline $\begin{array}{c}\text { F4. Damage associated with certain TDG accident } \\
\text { (assumption } \boldsymbol{j}) \\
\sum_{k}\left(P_{s c, i j k} \times \sum_{m}\left(F_{p, m} \times E_{i k m} \times S_{k m} \times\left(1-C_{f f, i k m}\right)\right)\right)\end{array}$ & $\begin{array}{l}\text { ADR traffic } \\
\text { planning and } \\
\text { emergency planning }\end{array}$ \\
\hline $\begin{array}{l}\text { F5. Magnitude of consequences (assumption } \boldsymbol{k} \text { ) } \\
\sum_{m}\left(F_{p, m} \times E_{i k m} \times S_{k m} \times\left(1-C_{f f, i k m}\right)\right)\end{array}$ & $\begin{array}{l}\text { Estimate of the } \\
\text { damage associated } \\
\text { with the evolution of } \\
\text { a specific } k \text { scenario }\end{array}$ \\
\hline $\begin{array}{l}\text { F6. Intrinsic road hazard rating } \\
\qquad P_{i s, i}\end{array}$ & $\begin{array}{l}\text { Road accident } \\
\text { prevention }\end{array}$ \\
\hline$\sum_{j}^{\text {F7. ADR flows }} P_{A D R, i j}$ & $\begin{array}{l}\text { ADR vehicle } \\
\text { quantification }\end{array}$ \\
\hline
\end{tabular}




\section{1 $\quad$ P $_{\text {is,i }}$ (intrinsic road hazard)}

The parameter $P_{\text {is,i }}$ represents the probability of each road elements to be interested by a generic road accident and it is expressed by means of an opportune accident rate indicator.

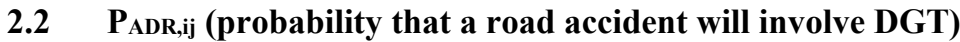

The quota of accident involving vehicles transporting dangerous goods (ADR vehicles) is unknown, so it is assumed that $\mathrm{P}_{\mathrm{ADR}}$ correspond to the ratio of traffic quota of dangerous goods transport to the overall traffic.

Real time monitoring through the gates monitoring network and OBU (On Board Units) placed on trucks will allow in the long term to improve the methodology with real data on ADR transits.

The orange license plate placed on all vehicles transporting dangerous goods is made of two numbers, the upper one (Kemler) identifying the hazard and the lower one (ONU) identifying the specific substance transported. There have been considered 60 hazard of interest and 12 model substances (Table 2) that represent the main incident scenarios. So the parameter $\mathrm{P}_{\mathrm{ADR}}$ is be a vector of 72 lines $(60+10)$ having different values for each road arch.

Table 2: Model substances.

\begin{tabular}{lll}
\hline $\begin{array}{l}\text { Kemler } \\
\text { number }\end{array}$ & $\begin{array}{l}\text { ONU } \\
\text { number }\end{array}$ & Substance \\
\hline $\mathbf{2 2 5}$ & 1073 & oxygen, refrigerated liquid \\
$\mathbf{2 2}$ & 1977 & nitrogen, refrigerated liquid \\
$\mathbf{2 0}$ & 1066 & nitrogen, compressed \\
$\mathbf{2 3}$ & 1049 & hydrogen, compressed \\
$\mathbf{2 3}$ & 1075 & petroleum gases, liquefied \\
$\mathbf{2 5}$ & 1072 & oxygen, compressed \\
$\mathbf{2 6 3}$ & 1040 & ethylen oxyde (+nitrogen) \\
$\mathbf{2 6 8}$ & 1005 & ammonia, anhydrous \\
$\mathbf{3 0}$ & 1202 & gas oil \\
$\mathbf{3 3}$ & 1203 & gasoline \\
$\mathbf{3 3 6}$ & 1230 & Methanol \\
$\mathbf{6 3}$ & 2023 & epichlorohydrin \\
\hline
\end{tabular}

\subsection{Psc,ijk (probability that a specific accident scenario with a known damage area and threshold will occur)}

$\mathrm{P}_{\mathrm{sc}, \mathrm{ijk}}$ is the probability that from a road accident involving an ADR vehicle a series of events determine a hazardous material release with a consequential scenario with a specific threshold and a specific damage area (buffer).

This parameter is a function of the probability of release, depending on the type of container used (atmospheric or pressure tank) and on the extent of the loss and the trigger probability. 
14 standard incident scenarios that can damage human and non human targets have been chosen and 27 scenario/substance combination have been identified.

\section{4 $E_{i k m}$ (potential exposed targets), $F_{p, m}$ (presence factor), $S$ (potential exposed targets susceptibility) and $\mathrm{C}_{\mathrm{ff}}$ (coping capacity)}

The parameter E express the potential targets exposed to the different standard incident scenarios.

The GIIS calculates and display the DGT risk for the targets listed in table 3.

Table 3: Human targets considered in the risk analysis model

\begin{tabular}{cc}
\hline Human Targets & Non-human targets \\
\hline Resident population & Structures \\
Industries employees & Woods \\
Hospital employees and users & Protected areas \\
School employees and users & Agricultural areas \\
Shopping center employees and users & Ground Waters \\
& Surface waters \\
\hline
\end{tabular}

As for human targets, $\mathrm{F}_{\mathrm{p}, \mathrm{m}}$ quantifies the fraction of potentially present targets as a proportion of those potentially exposed.

In the case of human targets, $\mathrm{F}_{\mathrm{p}, \mathrm{m}}$ is a necessary adimensional weight to sum up different exposed areas.

The parameter $\mathrm{S}_{\mathrm{km}}$ represents the fact that not all targets potentially exposed could be really damaged, whereas the $\mathrm{C}_{\mathrm{ff}, \mathrm{ikm}}$ is defined as the resilience or coping capacity, which means the capability of the territory to reduce the risk related to specific targets in specific accident scenario (e.g. the presence of firefighters detachments, time of arrival of an ambulance, presence of hospitals and evaluation of time taken to reach the nearest one, etc.).

$\mathrm{C}_{\mathrm{ff}, \mathrm{ikm}}$ is a factor that will reduce the amount of risk and the maximum value that can be assigned to it is 0.10 .

\section{GIIS functionalities and infrastructure}

Core of the GIIS is the preventive safety component, based on the highly detailed risk analysis model for land DGT. The GIIS is integrated with fixed monitoring gates, located in most critical areas, and is able to collect data from on-board units placed on vehicles of DGT companies that would like to be monitored in the future.

The great challenge of PTA-DESTINATION project is to improve the risk analysis model and optimizing the GIIS functionalities in order to increase its diffusion as a territorial governance tool, able to influence decision-making processes in urban planning, new infrastructures planning and management and route planning. 
Moreover accessibility to the tool will be enhanced by developing mobile Applications, extending the range of users.

System functionalities support the execution of elaborations differentiated on the basis of user profiles (VV.AA. [7]).

The solution is flexible enough to allow calculation of specific formulabranches and assessment of cumulate impact of multiple accident scenarios; moreover specific execution criteria (choice of geographic area, accident scenarios, human/environmental vulnerabilities...) are available for user selection, depending on chosen formula to be represented. Supported elaborations are the following:

- standard elaborations, available for all users, are referred to standard temporal and weather conditions; it's not possible for users to execute calculations changing the values or excluding/adding some of the formula parameters;

- custom elaborations, for advanced users, allow change (by amplifying or mitigating) of some parameter's values or introduction of correction factors taking into account time-dynamics (day/night rather than holiday/weekday) or weather specific conditions (rain/snow/fog);

- $\quad$ simulations, only for specialist users, are applied to potential scenarios, as they aim to simulate possible changes in the current context. The user can edit an additional target (e.g. presence of a shopping center, or a hospital) or manually enter new values for specific parameters of the risk formula.

Elaboration results are shown both in synthetic form (for each road-stretch), as shown in Figure 1, and in analytic form (Figure 2), individuating human and environmental vulnerabilities impacted by a specific accidental scenario.

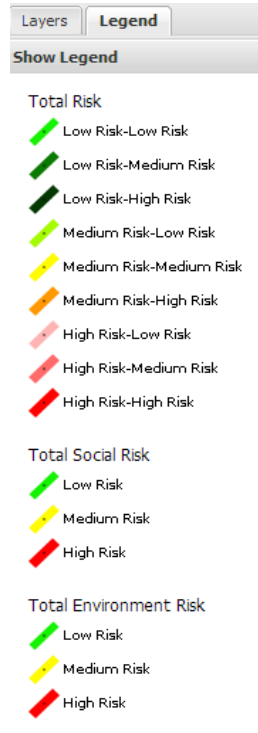

Figure 1: GIIS elaboration results synthetic view.

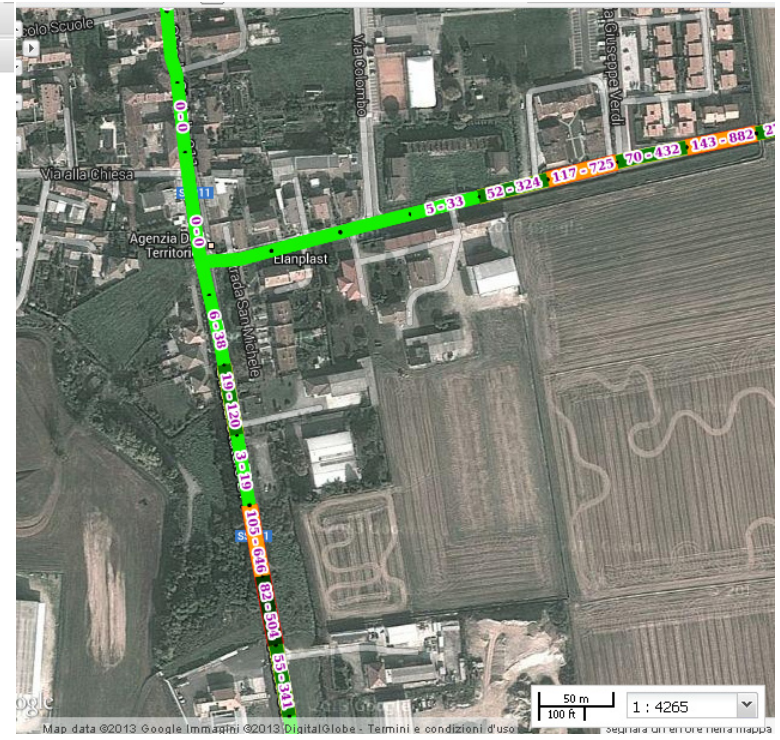




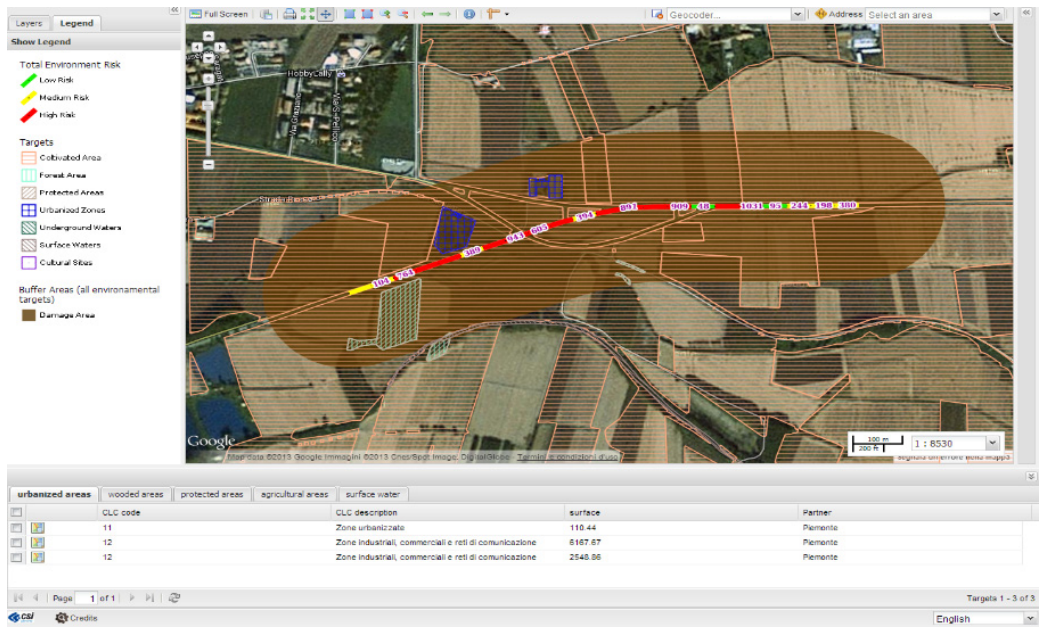

Figure 2: GIIS elaboration results analytic view.

Simulations results are showed in the analytic form box, where it is possible to edit a new target (Figure 3) or to modify parameters such as number of residents, number of employees, etc.

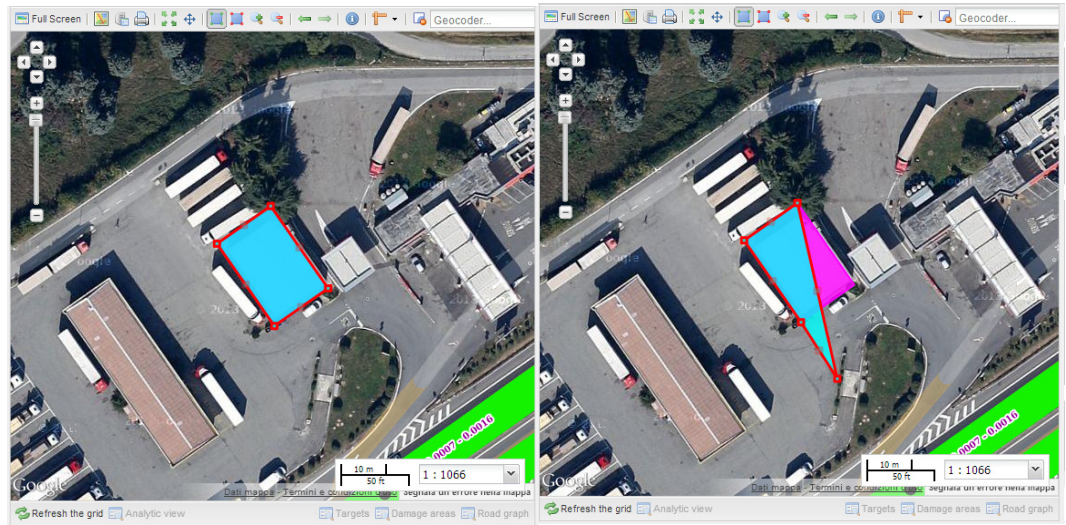

Figure 3: $\quad$ Shape editing - BEGIN and END.

As far as passive safety is concerned, it is possible to define impact and action areas, the potential risks caused by the domino effect and the anthropic and environmental damage and to assess the ability of responsible bodies to address the issue.

The infrastructure proposed is based on Open Source components in order to foster effectiveness through reuse of existing solutions as well as the possibility for both partner and external stakeholders to leverage on the software that will produce during the project lifetime. In order to ensure superior interoperability 
we will strive to adopt internationally recognized standards for the dissemination of the results via the infrastructure. Specifically we intend to leverage on the protocols mandated by OGC and ISO which compose the base building blocks for an INSPIRE compliant SDI, that is OGC Web Map Service (WMS), OGC Web Feature Service (WFS) as well as OGC Catalogue Service (CSW).

The main building blocks of the infrastructure are as follows:

- the storage system consists of a PostgreSQL/PostGIS database, for vector data management, and a shared file system, for raster data management;

- GeoServer (http://geoserver.org) provides all OGC standard geo-spatial services for all on the fly calculation requirements and any data extraction;

- GeoWebCache(http://geowebcache.org/), acting as an accelerator for the base maps, provides map tiling and caching tile services

- GeoBatch (http://geobatch.geo-solutions.it/) is an Open Source Java enterprise application developed by GeoSolutions, for the collection, processing and publication of geospatial data in real time. This component manages partners' data ingestion, and performs format and structure harmonization. Moreover GeoBatch automates all the preprocessing necessary for the formula calculations;

- MapStore (http://mapstore.geo-solutions.it/), a webgis Open Source framework developed by GeoSolutions, provides a multi-lingual user interface (Italian, French, English and soon German), integrating and orchestrating GeoServer OGC services and metadata explorer functionalities to browse the catalogues exposed in CSW format.

Sun Java Directory Server is responsible for providing credential storage functionalities. WSO2 Enterprise Service Bus (ESB) is going to provide MOM (Message Oriented Middleware) functionalities.

\section{Conclusions}

The above described functionalities are just the starting point for the further evolution of GIIS expected from PTA-DESTINATION project. Several are GIIS evolution and exploitation areas, here in conclusion are summarized the main focuses:

- $\quad$ exploiting monitoring data for estimating the flow of transport of ADR substances in the DGT risk formula, defining methodological criteria to optimize and automate the input for $\mathbf{P}_{\mathrm{ADR}, \mathbf{i j}}$ parameter in the formula;

- fine tuning of DGT risk methodology by refining of the use of the weighting factor $\left(\mathbf{F}_{\mathbf{p}, \mathbf{m}}\right)$ in relation to different accident scenarios and extension to specific ADR substances beyond model substances;

- implementation of apps for mobile devices aimed to representation of main GIIS output (DGT risk; ADR hazard, land vulnerability...) for citizen and to provide a damage assessment tool for immediate use to emergency managers (Fire Brigade, Police, Civil Protection, Environmental Agencies); 
- implementation of route planner functionalities enabling GIIS to calculation of best routes based on specific parameters DGT (DGT risk, ADR danger, etc.) in addition to traditional ones;

- implementation of use of voluntary detection devices for smartphone or tablet for DGT carriers not supporting OBUs systems. following:

The expected results from PTA-DESTINATION actions are the

- enhance preventive safety functionalities, by taking advantage of information gathered by monitoring network (active safety);

- $\quad$ supporting more flexible custom elaborations, taking into account actual specific characteristics of areas from the point of view of both factors of danger and vulnerability;

- $\quad$ disseminating PTA-DESTINATION outputs, conveying them to citizen in simplified/aggregated mobile applications to use the GIIS in preventive safety and as a support tool for the active and passive safety;

- $\quad$ expand the number of potential GIIS users as real time tool for PAs and transporters;

- $\quad$ ensuring integration of GIIS with info-mobility tools.

Beyond PTA-DESTINATION project actions, flexibility of GIIS solution could allow to consider dangerous good transport risk assessment (actual GIIS outputs) as a first prototype in the standpoint of extending the solution to support an overall sustainability assessment both at strategic and at local level. Obviously a strong methodological effort is required to run this path, but GIIS solution has been designed to be applied to different thematic areas.

\section{References}

[1] M. Benza, C. Bersani, R. Sacile, A. Tomasoni \& E. Trasforini, A distributed information system prototype to detect and monitor the Hazardous Material Transport on the road in the territory of Nice-Imperia-Ventimiglia WIT Transactions on The Built Environment, Vol 94, 327-336, WIT Press $<$ www.witpress.com $>$ accessed 30.11.2011, ISSN 1743-3509 (on-line), DOI:10.2495/SAFE070321, 2007.

[2] G. Pastorelli, Integrated Risk Area Planning: The case of Milan Metropolitan Area, Dangerous materials: Control, Risk prevention and crisis management, NATO Science for Peace and Security Series C: Environmental Security, Volume 00, Part 3, 147-164, DOI: 10.1007/978-90-481-9732-3_13, 2010.

[3] ADR 2013 European Agreement concerning the International Carriage of Dangerous Goods by Road, United Nations Economic Commission for Europe, ECE/TRANS/225, New York and Geneva, 2012.

[4] VV.AA., Destination. Man, road, environment. Only one destination. Vol. 1: Project Overview, Regione Piemonte, ISBN 978-88-98878-02-4, 2014. 
[5] VV.AA., Destination. Man, road, environment. Only one destination. Vol. 3: Monitoring Network, Regione Piemonte, ISBN 978-88-98878-07-9, 2014.

[6] VV.AA., Destination. Man, road, environment. Only one destination. Vol. 2: TMP risk analysis model (summary), Regione Piemonte, ISBN 97888-98878-06-2, 2014.

[7] VV.AA., Destination. Man, road, environment. Only one destination. Vol. 4: GIIS - Global Integrated Information System (summary), Regione Piemonte, ISBN 978-88-98878-13-0, 2015. 